Lymphology 53 (2020) 20-28

\title{
CYP26B1 AND ITS IMPLICATIONS IN LYMPHANGIOGENESIS: LITERATURE REVIEW AND STUDY OF RARE VARIANTS IN TWO FAMIIUIES
}

M. Ricci, R. Serrani, B. Amato, R. Compagna, D. Veselenyiova, S. Kenanoglu, D. Kurti, M. Baglivo, J. Krajcovic, G.A.D. Miggiano, B. Aquilanti, G. Matera, V. Velluti, L. Gagliardi, M. Dundar, S.H. Basha, M. Bertelli

Division of Rehabilitation Medicine (MR,RS), Azienda Ospedaliero-Universitaria, Ospedali Riuniti di Ancona, Italy; Department of General and Geriatric Surgery (BA), University of Naples "Federico II", Naples, Italy; Department of Public Health (RC), University of Naples Federico II, Naples, Italy; Department of Biology (DV,JK), Faculty of Natural Sciences, University of Ss. Cyril and Methodius In Trnava, Trnava, Slovakia; MAGI Euregio (DV,SK,DK,MBa,MBe), Bolzano, BZ (headquarters); San Felice del Benaco (branch), Italy; Research Center in Human Nutrition (GADM), Sacro Cuore Catholic University, Rome, Italy; UOC Clinical Nutrition (BA,GM,VV,LG), Department of Medical Genetics, Faculty of Medicine, Erciyes University, Kayseri, Turkey, Policlinico Universitario Foundation A. Gemelli IRCCS, Rome, Italy; Department of Medical Genetics (SK,MD), Faculty of Medicine, Erciyes University, Kayseri, Turkey; MAGI-Balkan (MBe), Tirana, Albania; Innovative Informatica Technologies (SHB), Hyderabad, India; EBTNA-Lab (MBe), Rovereto, TN, (headquarters), San Felice del Benaco, Italy; MAGI's Lab (MBe), Rovereto, TN, (headquarters); San Felice del Benaco, Italy

\section{ABSTRACT}

CYP26B1 is a member of the cytochrome P450 family and is responsible for the breakdown of retinoic acid forwhich appropriate levels are important for normal development of the cardiovascular and lymphatic systems. In a cohort of 235 patients with lymphatic malformations, we performed genetic testing for the CYP26B1 gene. These probands had previously tested negative for known lymphedema genes. We identified two heterozygous missense CYP26B1 variants in two patients. Our bioinformatic study suggested that alterations caused by these variants have no major effect on the overall stability of CYP26B1 protein structure. Balanced levels of retinoic acid maintained by CYP26B1 are crucial for the lymphatic system. We identified that CYP26B1 could be involved in predisposition for lymphedema. We propose that CYP26B1 be further explored as a new candidate gene for genetic testing of lymphedema patients.

Keywords: CYP26B1 (Cytochrome P450 Family 26 Subfamily B Member 1), NGS (Next Generation Sequencing), lymphedema, genetic diagnosis, lymphvascular genomics, lymphangiogenesis, retinoic acid

CYP26B1 is a gene that encodes for CYP26B1 (Cytochrome P450 Family 26 Subfamily B Member 1), also known as P450RAI2. It is one of three isoforms of CYP26 (CYP26A1, CYP26B1 and CYP26C1) identified in humans (1-3). The gene consists of eight exons and covers about $18 \mathrm{kbp}$. It also includes a 3 'UTR of almost $3 \mathrm{~kb}$ (4). The CYP26B1 gene maps to 2 p13.2; after transcription and translation, CYP26B1 protein is transported to the 
endoplasmic reticulum. CYP26B1 is a monooxygenase responsible for the synthesis of cholesterol, steroids and other lipids. It plays an important role in cell proliferation, differentiation, and apoptosis and in many developmental processes.

The lymphatic vasculature is an essential component of the lymphatic system. It is composed of lymphatic capillaries, pre-collecting lymphatic vessels, and collecting lymphatic vessels (5). Lymphatic vessels drain interstitial fluid, lipids, and other macromolecules and collaborate with the immune system. Fluid collected by the lymphatic system is delivered to the lymph nodes and/or returned to the blood circulatory system (6). Impairment of this balanced homeostasis can cause disorders such as lymphedema and other inflammatory diseases (7).

Lymphedema is a disorder caused by dysfunction of the lymphatic system. It can be caused by impaired function of lymph nodes or malformation of lymphatic vessels (LVs). It leads to a blockage of the flow of lymphatic fluid and fluid build-up in tissues (8). Proper development and function of LVs is therefore important.

Lymphatic capillaries are specialized vessels surrounded by a discontinuous basement membrane and cell junctions that permit entry of fluid and immune cells (9). It is unclear how they are formed. Animal models, such as mice and zebrafish embryos, provided the insights that LVs are formed from lymphatic endothelial cells (LECs), which differentiate at least in large part from the cardinal and embryonic veins $(10,11)$. Lymphatic vessels differ from blood vessels under normal conditions in lacking perivascular cells like pericytes and vascular smooth muscle cells although these features may be acquired in pathologic states. There is nonetheless a clear connection between the development of lymphatic vessels and blood vessels. CYP26B1 plays a role in both.

Retinoic acid (RA) is an active derivative of vitamin A (retinol, ROH), synthesized by retinaldehyde dehydrogenase (RALDH) (12) and degraded by members of the cytochrome P450 family 26 (CYP26s) (Fig. 1) $(13,14)$. Retinol is a crucial regulator of gene expression in biological processes such as cell proliferation and differentiation. Appropriate levels of RA are important for development of the cardiovascular and lymphatic systems and other organs. Retinoic acid levels in the body are regulated by various enzymes, including CYP26B1 (15). CYP26B1 plays a key role in the regulation of retinoid levels in vascularization processes (15).

The importance of RA in lymphatic vascular development was demonstrated by Bowles and colleagues (16), who detected RA in the cardinal vein of a mouse model and showed that lower signaling activity of RA is associated with LEC progenitors (16). This finding suggests that RA levels might need to be low to ensure appropriate development of LECs from their LEC progenitors. Choi et al showed that RA promotes proliferation and migration of LECs and therefore lymphangiogenesis in a mouse model (17). They also demonstrated that 9-cis-RA has positive effects on lymphatic vessel regeneration (17).

Higher levels of RA have been reported in Cyp26b1-null mice. Silencing of Cyp26b1 abolished the machinery necessary for breakdown of RA, leading to constantly elevated levels of RA. The number of lymphatic endothelial progenitor cells subsequently increased in the cardinal vein. Other lymphatic abnormalities were also observed, including hyperplastic bloodfilled lymph sacs and hyperplastic dermal lymphatic vessels (16). Other animal models have also been used to study CYP26B1. High RA levels in Xenopus laevis embryos are associated with excessive lymphangiogenesis, which may lead to obstruction of lymph flow (18).

Human polymorphisms in CYP26B1 have been associated with different diseases. Fransen et al reported an association between a CYP26B1 variant and Crohn's disease. This chronic inflammation of the gut has been linked to vitamin A metabolism. The authors showed that probands homozygous for $\mathrm{CY}$ $P 26 B 1$ variant rs2241057 are at higher risk of developing Crohn's disease because retinoic 


\begin{tabular}{|c|c|c|c|c|}
\hline \multicolumn{5}{|c|}{$\begin{array}{c}\text { TABLE } 1 \\
\text { Human Phenotype Associated with CYP26B1 }\end{array}$} \\
\hline Gene & Function & OMIM disease & Inheritance & Lymphatic phenotype \\
\hline$C Y P 26 B 1$ & $\begin{array}{l}\text { Protein } \\
\text { coding }\end{array}$ & $\begin{array}{l}\text { 614416, Craniosynostosis with } \\
\text { radiohumeral fusions and } \\
\text { other skeletal and craniofacial } \\
\text { anomalies }\end{array}$ & $\begin{array}{l}\text { Autosomal } \\
\text { recessive }\end{array}$ & $\begin{array}{l}\text { Dysregulation of RA, high } \\
\text { levels of RA, excessive } \\
\text { lymphangiogenesis }{ }^{16,20}\end{array}$ \\
\hline
\end{tabular}

TABLE 2

Primary Amino Acid Sequence for Which Templates were Searched and for Which Models were Built

MLFEGLDLVSALATLAACLVSVTLLLAVSQQLWQLRWAATRDKSCKLPIPKGSMGFPLIGETGH WLLQGSGFQSSRREKYGNVFKTHLLGRPLIRVTGAENVRKILMGEHHLVSTEWPRSTRMLLGP NTVSNSIGDIHRNKRKVFSKIFSHEALESYLPKIQLVIQDTLRAWSSHPEAINVYQEAQKLTFRM AIRVLLGFSIPEEDLGHLFEVYQQFVDNVFSLPVDLPFSGYRRGIQARQILQKGLEKAIREKLQCT QGKDYLDALDLLIESSKEHGKEMTMQELKDGTLELIFAAYATTASASTSLIMQLLKHPTVLEKL RDELRAHGILHSGGCPCEGTLRLDTLSGLRYLDCVIKEVMRLFTPISGGYRTVLQTFELDGFQIP KGWSVMYSIRDTHDTAPVFKDVNVFDPDRFSQARSEDKDGRFHYLPFGGGVRTCLGKHLAKL FLKVLAVELASTSRFELATRTFPRITLVPVLHPVDGLSVKFFGLDSNQNEILPETEAMLSATV

acid is not effectively catabolized in these individuals. This defect leads to accumulation of RA and promotion of lymphangiogenesis and inflammation. In contrast, the absence of this allele is linked to protection against inflammation (19).

Another CYP26B1 polymorphism has been implicated in atherosclerosis or chronic inflammatory disease of the blood vessels. Polymorphism rs2241057 was found to affect CYP26B1-regulated levels of retinoids and possibly the development of atherosclerosis (20). Since the development of blood and lymphatic vessels is closely linked, it is reasonable to speculate that polymorphisms in CYP26B1 may also affect lymphatic vessel development and function (Table 1).

\section{MATERIALS AND METHODS}

\section{Clinical Evaluations}

We analyzed samples from 246 Caucasian patients diagnosed with primary lymphedema in hospitals across Italy. The patients were included retrospectively in our study. No consanguinity was reported in the families. Clinical diagnosis of lymphedema was according to generally approved clinical criteria. DNA was extracted from saliva or blood of probands for genetic testing. All data were collected as part of routine diagnosis.

Genetic Analysis

A custom-made oligonucleotide probe library was designed to capture all coding exons and flanking exon/intron boundaries $( \pm 15 \mathrm{bp})$ of 29 genes known to be associated with lymphedema. Our panel included the candidate gene CYP26B1. Variants with likely clinical significance identified in DNA of probands were confirmed by bidirectional Sanger sequencing on a CEQ8800 Sequencer (Beckman Coulter).

We searched the international databases dbSNP and Human Gene Mutation Database Professional for all nucleotide changes. In silico evaluation of the pathogenicity of changes in the sequence of CYP26B1 was performed using the Variant Effect Predictor tool and MutationTaster. Minor allele frequencies (MAF) were checked in the Genome Aggregation Database (gnomAD). All variants were evaluated according to American College of Medical Genetics and Genomics guidelines (21). Detailed pre-test genetic counseling was provided to all subjects, who were then invited to sign specific informed consent according to Institutional Review Board guidelines to use their anonymized genetic results for research. 


\begin{tabular}{|c|c|c|c|l|}
\hline \multicolumn{7}{|c|}{ Clinical Features of Female Probands with CYP26B1 Variants } \\
\hline Age & Clinical features & $\begin{array}{c}\text { Age of } \\
\text { onset }\end{array}$ & $\begin{array}{c}\text { Family history of } \\
\text { lymphedema }\end{array}$ & \multicolumn{1}{|c|}{ Variant nomenclature } \\
\hline 55 & $\begin{array}{c}\text { lower limb } \\
\text { lymphedema, nanism }\end{array}$ & infancy & NO & $\begin{array}{l}\text { NM_001277742.1:c.37G }>\text { A } \\
\text { p.(Ala13Thr) }\end{array}$ \\
\hline 55 & left lower limb edema & 48 years & NO & $\begin{array}{l}\text { NM_001277742.1:c.1284G }>\text { T } \\
\text { p.(Glu428Asp) }\end{array}$ \\
\hline
\end{tabular}

\begin{tabular}{|c|c|c|c|c|c|}
\hline \multicolumn{7}{|c|}{ TABLE 4 } \\
\hline Vharacterization of the Identified CYP26B1 Variants \\
\hline Variants & dbSNP & Varsome & $\begin{array}{c}\text { Mutation } \\
\text { Taster }\end{array}$ & Polyphen & Frequency \\
\hline +NM_001277742.1:c.37G>A & $/$ & $\begin{array}{c}\text { Likely } \\
\text { Benign }\end{array}$ & $\begin{array}{c}\text { disease } \\
\text { causing }\end{array}$ & $\begin{array}{c}\text { Potentially } \\
\text { damaging }\end{array}$ & $/$ \\
\hline NP_001264671.1:p.Ala13Thr & & $\begin{array}{c}\text { Likely } \\
\text { Benign }\end{array}$ & $\begin{array}{c}\text { disease } \\
\text { causing }\end{array}$ & benign & 0.00003 \\
\hline NP_001264671.1:p.Glu428Asp
\end{tabular}

In Silico Analysis

The primary amino acid sequence of CYP26B1 in FASTA format (Table 2) was used to search for template libraries in Swiss model template library (SMTL) version 201910-24 and Protein Data Bank (PDB) release 2019-10-18 (22) with BLAST (Basic Local Alignment Search Tool) (23) and HHBlits (24) for evolution-related structures matching the given CYP26B1 sequence. Models based on target-template alignment were built using ProMod3 of the SWISS-MODELING server (25). Coordinates conserved between the target and the template were copied from the template to the model. Insertions and deletions were remodeled using a fragment library. Side chains were then rebuilt. Finally, the geometry of the resulting model was regularized using the CHARMM27 force field (26). If loop modeling with ProMod3 failed, an alternative model was built with PROMOD-II (27). Global and per-residue model quality was assessed using the QMEAN scoring function (28). BioVia Discovery Studio Visualizer ver17.2 (29) was used to visualize the modeled protein, to mutate the targeted amino acids and to analyze molecular level interactions.
RESULTS

\section{Clinical and Genetic Assessment}

Of the 246 samples, 235 tested negative for known lymphedema genes. Negative samples underwent a second test for possible new candidate genes, including CYP26B1. Two patients with two heterozygous variants in the CYP26B1 gene were identified. Both cases were sporadic and had no family history of lymphedema. Table 3 shows the clinical features of these patients.

The first female proband was diagnosed with lymphedema in infancy and showed lymphedema of the. lower limbs and restricted growth. The missense variant NM_001277742.1:c.37G >A was identified. It has no dbSNP ID and allele frequency is unknown. PolyPhen describes this variant as possibly damaging (Table 4).

The second female proband showed left lower limb edema that manifested at age 48 years. The missense CYP26B1 variant was identified as NM_001277742.1:c.1284G > T. It is characterized in dbSNP as rs202200784 and has a total frequency of 0.00003 according to GnomAD. As shown in , two out of three predictors (Mutation Taster and Polyphen) report the variants as potentially damaging. Both 


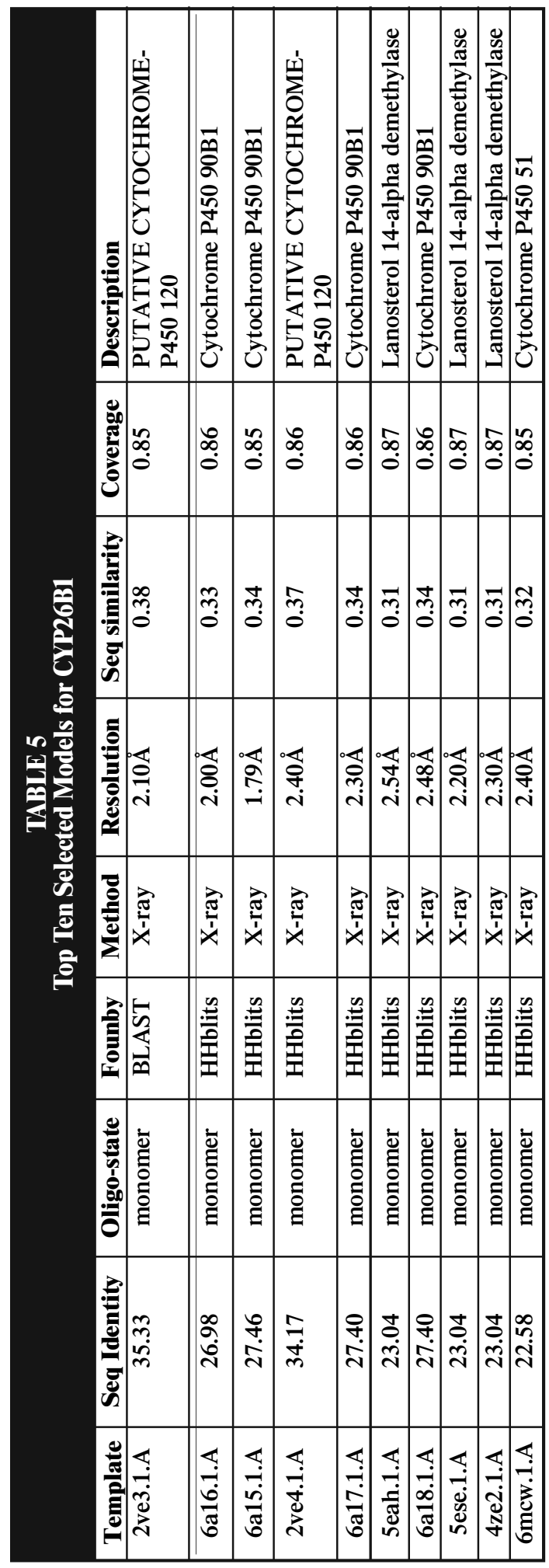

variants are reported on Varsome as Likely Benign, with BP1 and BP4 supporting criteria.

\section{In Silico Analysis, Template Selection and Model Building}

Template search with BLAST and HHBlits was performed against the SWISS-MODEL template library (SMTL, last update: 2019-10-24, last included PDB release: 2019-10-18). The target sequence was searched with BLAST against the primary amino acid sequence in SMTL. A total of 767 templates matched the target sequence with varied sequence identity and quality percentages. Details of the top 10 templates are shown in Table 5.

The 2ve3.1.A template was selected on the basis of sequence identity and similarity to align and query sequences for model building. The resulting model is shown in Fig. 1. The Discovery studio visualizer was used to generate Glu428Asp and Ala13Thr mutated structural versions. Molecular level interaction analysis was conducted between native/mutated residues with residue interactions. Snapshots are shown in Fig. 2a,b. Details of the residues involved in interactions, along with the type of bonds they formed and bond lengths in angstrom units, are shown in Tables 6 and 7, respectively. While there is no difference on length of the bond with the interacting residue for Ala13 and Thr13 (Table 6), the length of the bond between Lys444 and the variants is greater (Table 7).

\section{DISCUSSION AND CONCLUSION}

Lymphedema is a chronic disease involving brawny edema, inflammation, and fibrosis due to accumulation of high protein interstitial fluid (8). The incidence of primary lymphedema is approximately at least 1 in 100,000 individuals (30). Despite recent advances, the genetics of lymphedema is still not fully understood. The discovery of new genes with a role in lymphatic system development and/or associated with lymphedema or its predisposi- 


\begin{tabular}{|c|c|c|c|c|}
\multicolumn{5}{c}{$\begin{array}{c}\text { TABLE } 6 \\
\text { Details of Molecular Interactions of ALA13 and THR13 of the } \\
\text { Modeled CYP26B1 Protein with Adjacent Residues }\end{array}$} \\
\hline \multirow{2}{*}{ Mutation } & Amino acid & $\begin{array}{c}\text { Molecular interactions } \\
\text { observed }\end{array}$ & $\begin{array}{c}\text { Bond length in } \\
\text { angstroms }\end{array}$ & Bond type \\
\hline \multirow{2}{*}{ Ala13Thr } & Ala13 & ALA17:N - ALA13:O & 2.93 & H-bond \\
\cline { 2 - 5 } & Thr13 & ALA17:N - THR13:O & 2.93 & H-bond \\
\hline
\end{tabular}

\begin{tabular}{|c|c|c|c|c|}
\hline \multicolumn{5}{c|}{$\begin{array}{c}\text { TABLE } 7 \\
\text { Details of Molecular Interactions of a) GLU428 and b) ASP428 of the } \\
\text { Modeled CYP26B1 Protein with Adjacent Residues }\end{array}$} \\
\hline \multirow{2}{*}{ Mutation } & Amino acid & $\begin{array}{c}\text { Molecular interactions } \\
\text { observed }\end{array}$ & $\begin{array}{c}\text { Bond length in } \\
\text { angstroms }\end{array}$ & Bond type \\
\hline \multirow{3}{*}{ Glu428Asp } & \multirow{2}{*}{ Glu428 } & LYS444:N - GLU428:O & 5.14 & H-bond \\
\cline { 2 - 5 } & \multirow{2}{*}{ Asp 428 } & SER422:C - GLU428:O & 3.22 & H-bond \\
\cline { 2 - 5 } & & LYS444:N - ASP428:O & 5.59 & H-bond \\
\cline { 2 - 5 } & & SER422:C - ASP428:O & 3.22 & H-bond \\
\hline
\end{tabular}

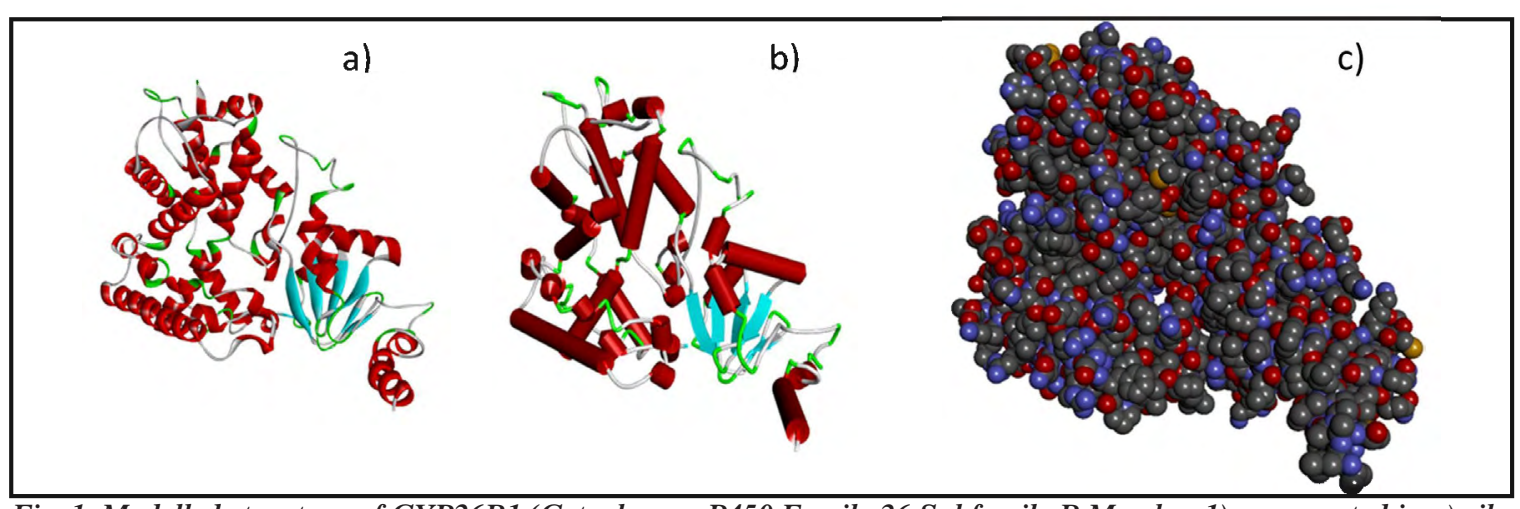

Fig. 1. Modelled structure of CYP26B1 (Cytochrome P450 Family 26 Subfamily B Member 1) represented in a) ribbon b) schematic and c) CPK view. Cyan colored regions represent beta sheets, white regions represent loops and red regions represent alpha helices. Carbon is represented with colored grey, Oxygen is red, Nitrogen is blue, Hydrogen is white, Chlorine is green, Sulfur is yellow, Phosphorus is orange, and Iron is brown.

tion is therefore important.

We tested 246 primary lymphedema patients for known genes associated with the disease (31). We obtained negative results for 235 samples. These were subsequently tested for possible candidate genes, including CYP26B1. We found variants in the CYP26B1 gene in two of the samples. Both variants were heterozygous missense mutations. One of the variants is listed in dbSNP (rs202200784) with a reported frequency of 0.00003 . Both female probands were sporadic cases. No other family members were tested.

In silico analysis of the effect of the variants on the overall structure of CYP26B1 protein demonstrated that gene structure coded with Ala13 has no major difference in stability with respect to mutated Thr13 in terms of direct hydrogen bonding with nearby Ala17. Both bonds have the same bond length of 2.93 Angstroms (Table 6). The same was observed for Glu428Asp, where two direct hydrogen bonds with Lys444 and Ser422 were observed. However, Glu428 formed a slightly stronger 
hydrogen bond with Lys144 (5.14 ̊) than did Asp428 (5.59 ̊) (Table 7). These results suggest that the overall conformation of the protein might be somehow altered by these different interactions with nearby residues, leading to functional defects in the protein (Fig. 3).

To our knowledge, no association between CYP26B1 and lymphedema has ever been reported. The $C Y P 26 B 1$ gene is mostly studied in the context of bone development and skeletal
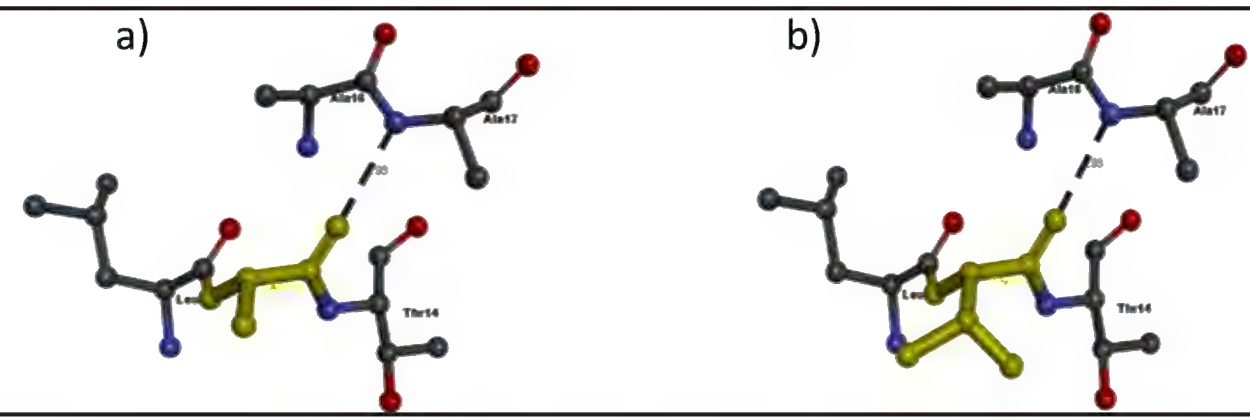

Fig. 2. Molecular interactions of a) ALA13 and b) THR13 (highlighted in yellow) of the modeled CYP26B1 protein with adjacent residues.

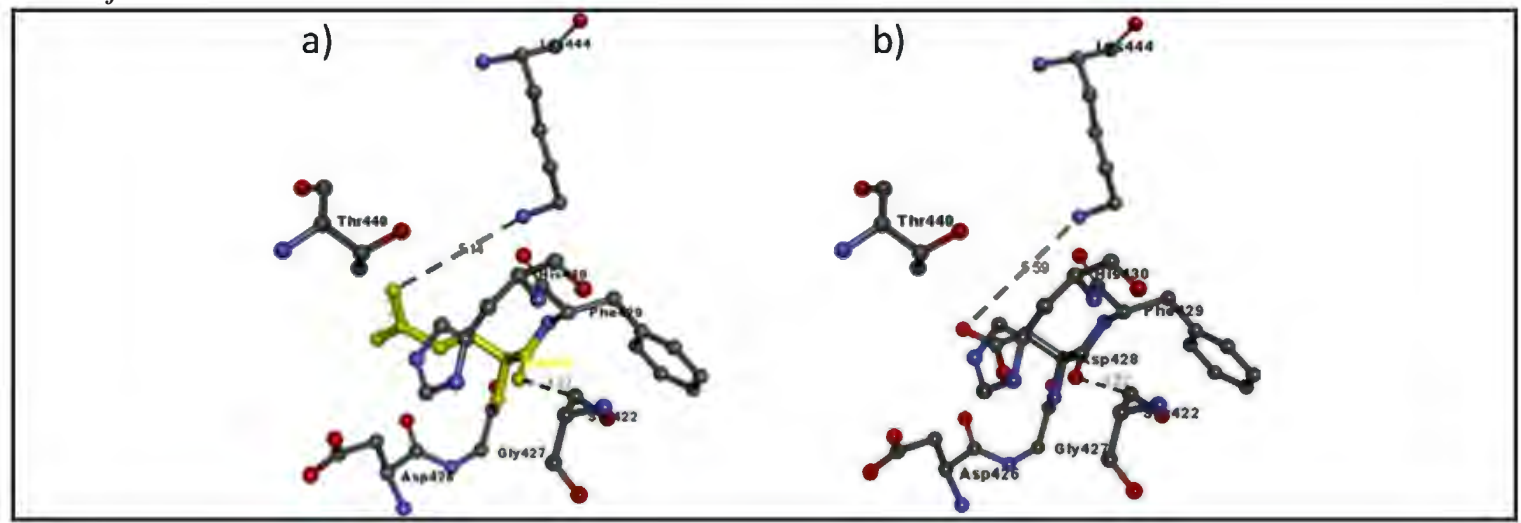

Fig. 3. Molecular interactions of a) GLU428 and b) ASP428 (highlighted in yellow) of the modeled CYP26B1 protein with adjacent residues

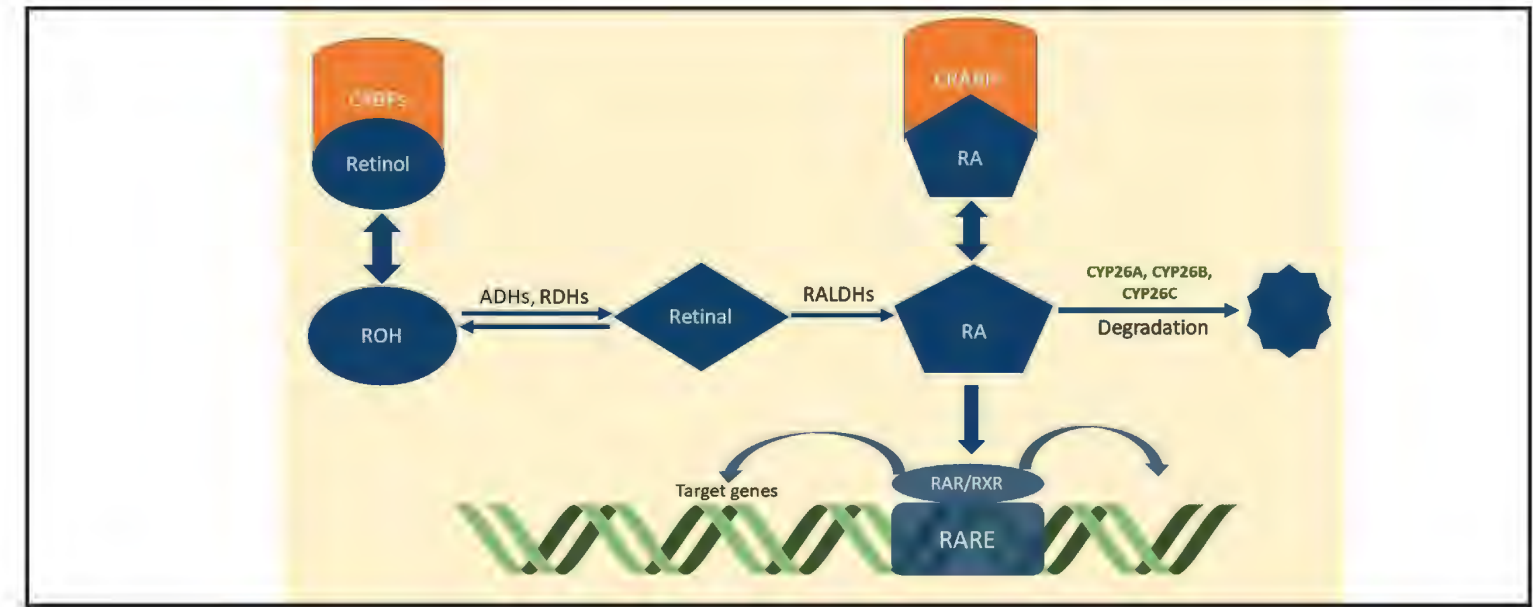

Fig. 4. The metabolism of retinoic acid: Vitamin A (retinol, $\mathrm{ROH}$ ) is hydrolyzed by alcohol dehydrogenase ADH and retinol dehydrogenase $R D H$ to retinal. Retinal is then oxidized by retinaldehyde dehydrogenase RALDH, creating retinoic acid, the active form of vitamin $A$. RA is degraded by enzymes of CYP26 family, mainly CYP26B1 (35). 
malformations. Laue et al reported a homozygous loss-of-function mutation in CYP26B1 that causes multiple skeletal defects, long-bone fusions and craniosynostosis in humans (32).

A GWAS led by Kichaev and colleagues (GWAS catalog GCST007841) investigated an association between CYP26B1 variants and body height (33). One of our probands displayed restricted growth, which might be related to the missense variant we identified.

However, the literature also reports a regulatory role of CYP26B1 in the metabolism of retinoic acid (Fig. 4), levels of which are linked directly to lymphangiogenesis $(13,15,16)$. Excessive lymphangiogenesis causes overgrowth of lymphatic vessels, which can lead to lymphedema by impeding the flow of lymph and causing it to accumulate in the tissues (5). CYP26B1 has potential not only as a candidate gene for genetic testing but perhaps also as a therapeutic target. Increasing the degradation of RA in patients with high levels of RA might potentially resolve inordinate lymphangiogenesis and prevent excessive formation of lymphatic vessels, establishing normal lymphatic vessel development.

In conclusion, in this article we review the scientific literature on the relationship between regulation of retinoic acid metabolism by $\mathrm{CY}$ P26B1 and development of lymphatic vessels from their LEC progenitors. We suggest that CYP26B1 might play a significant role in the development of malformations of the lymphatic vessels and in predisposition for lymphedema. Targeted NGS is indeed the best approach to determine genotype-phenotype correlations, segregation and recurrence risk in families, and improvement of diagnostic techniques is needed to offer patients more targeted drugs to treat their lymphatic disorders (34). On the basis of variants described in this paper, we suggest inclusion of CYP26B1 in the NGS gene panels for testing patients suffering from lymphatic system malformations and lymphedema.

\section{CONFLICT OF INTEREST AND \\ DISCLOSURE}

The authors declare no competing financial interests exist.

\section{REFERENCES}

1. Taimi, M, C Helvig, J Wisniewski, et al. A Novel Human Cytochrome P450, CYP26C1, Involved in metabolism of 9-cis and all-trans isomers of retinoic acid. J. Biol. Chem. 279 (2004), 77-85. doi:10.1074/jbc.M308337200

2. Pavez Loriè, E, H Li, A Vahlquist, H Törmä: The involvement of cytochrome p450 (CYP) 26 in the retinoic acid metabolism of human epidermal keratinocytes. Biochim. Biophys. Acta - Mol. Cell Biol. Lipids 179 (2009), 220-228. doi:10.1016/j. bbalip.2008.12.004

3. Zolfaghari, R, CJ Cifelli, SO Lieu, et al: Lipopolysaccharide opposes the induction of CYP26A1 and CYP26B1 gene expression by retinoic acid in the rat liver in vivo. Am. J. Physiol. - Gastrointest. Liver Physiol. 292 (2007), 1029-1037. doi:10.1152/ ajpgi.00494.2006

4. Elmabsout, AA, A Kumawat, P Saenz-Méndez, et al. Cloning and functional studies of a splice variant of CYP26B1 expressed in vascular cells. PLoS One 7 (2012), 1-8. doi:10.1371/journal. pone.0036839

5. Pichol-Thievend, C, BM Hogan, M Francois: Lymphatic vascular specification and its modulation during embryonic development. Microvasc. Res. 96 (2014), 3-9. doi:10.1016/j.mvr.2014.07.011

6. Foldi, M: Lymph formation and lymph flow: Physiological lymph drainage. In: Foundations of Manual Lymph Drainage. Elsevier; 2005:28-37. doi:10.1016/B978-032303064-9.50008-6

7. Alitalo, K: The lymphatic vasculature in disease. Nat. Med. 17 (2011), 1371-1380. doi:10.1038/ nm.2545

8. Michelini, S, M Cardone, P Maltese, et al: Primary lymphedema and genetic implications. EuroBiotech. J. 1(s2) (2017), 144-146. doi:10.24190/ ISSN2564-615X/2017/S2.07

9. Pawlikowski, B, J Wragge, JA Siegenthaler: Retinoic acid signaling in vascular development. Genesis. 57 (2019), e23287. doi:10.1002/dvg.23287

10. Srinivasan, RS, ME Dillard, OV Lagutin, et al: Lineage tracing demonstrates the venous origin of the mammalian lymphatic vasculature. Genes Dev. 21 (2007), 2422-2432. doi:10.1101/ gad.1588407

11. Yaniv, K, S Isogai, D Castranova, et al: Live imaging of lymphatic development in the zebrafish. Nat. Med. 12 (2006), 711-716. doi:10.1038/nm1427 
12. Niederreither, $\mathrm{K}, \mathrm{J}$ Vermot, $\mathbf{N}$ Messaddeq, et al: Embryonic retinoic acid synthesis is essential for heart morphogenesis in the mouse. Development 128 (1999), 1019-1031.

13. Abu-Abed, S, P Dollé, D Metzger, et al: The retinoic acid-metabolizing enzyme, CYP26A1, is essential for normal hindbrain patterning, vertebral identity, and development of posterior structures. Genes Dev. 15 (2001), 226-240. doi:10.1101/ gad.855001

14. Sakai, Y, C Meno, H Fujii, et al: The retinoic acid-inactivating enzyme CYP26 is essential for establishing an uneven distribution of retinoic acid along the anterio-posterior axis within the mouse embryo. Genes Dev. 15 (2001), 213-225. doi:10.1101/gad.851501

15. Ocaya, PA, AA Elmabsout, PS Olofsson, et al: CYP26B1 plays a major role in the regulation of all-trans-retinoic acid metabolism and signaling in human aortic smooth muscle cells. J. Vasc. Res. 48 (2010), 23-30. doi:10.1159/000317397

16. Bowles, J, G Secker, C Nguyen, et al: Control of retinoid levels by CYP26B1 is important for lymphatic vascular development in the mouse embryo. Dev. Biol. 386 (2014), 25-33. doi:10.1016/j. ydbio.2013.12.008

17. Choi, I, S Lee, HK Chung, et al: 9-Cis retinoic acid promotes lymphangiogenesis and enhances lymphatic vessel regeneration therapeutic implications of 9-Cis retinoic acid for secondary lymphedema. Circulation 125 (2012), 872-882. doi:10.1161/circulationaha.111.030296

18. Marino, D, V Dabouras, AW Brändli, et al: A role for all-trans-retinoic acid in the early steps of lymphatic vasculature development. J. Vasc. Res. 48 (2011), 236-251. doi:10.1159/000320620

19. Fransen, K, P Franzén, A Magnuson, et al: Polymorphism in the retinoic acid metabolizing enzyme CYP26B1 and the development of Crohn's disease. PLoS One 8 (2013), 1-5. doi:10.1371/journal.pone.0072739

20. Krivospitskaya, O, AA Elmabsout, E Sundman, et al: A CYP26B1 polymorphism enhances retinoic acid catabolism and may aggravate atherosclerosis. Mol. Med. 18 (2012), 712-718. doi:10.2119/ molmed.2012.00094

21. Richards, S, N Aziz, S Bale, et al: Standards and guidelines for the interpretation of sequence variants: A joint consensus recommendation of the American College of Medical Genetics and Genomics and the Association for Molecular Pathology. Genet. Med. (2015). doi:10.1038/gim.2015.30

22. Berman, HM, J Westbrook, Z Feng, et al: The Protein Data Bank. Nucleic Acids Res. 28 (2000), 235-242. doi:10.1093/nar/28.1.235

23. Camacho, C, G Coulouris, V Avagyan, et al: BLAST+: Architecture and applications. BMC Bioinformatics 10 (2009), 1-9. doi:10.1186/14712105-10-421
24. Remmert, M, A Biegert, A Hauser, et al: Lightning-fast iterative protein sequence searching by HMM-HMM alignment. Nat. Methods 9 (2012), 173-175. doi:10.1038/nmeth.1818

25. Waterhouse, A, M Bertoni, S Bienert, et al: SWISS-MODEL: Homology modelling of protein structures and complexes. Nucleic Acids Res. 46 (2018), W296-W303. doi:10.1093/nar/gky427

26. Mackerell, AD, M Feig, CL Brooks: Extending the treatment of backbone energetics in protein force fields: Limitations of gas-phase quantum mechanics in reproducing protein conformational distributions in molecular dynamics simulation. $\mathbf{J}$. Comput. Chem. 25 (2004), 1400-1415. doi:10.1002/ jcc. 20065

27. Guex, N, MC Peitsch, T Schwede: Automated comparative protein structure modeling with SWISS-MODEL and Swiss-PdbViewer: A historical perspective. Electrophoresis. 30(SUPPL. 1) (2009), 62-173. doi:10.1002/elps.200900140

28. Benkert, P, M Biasini, T Schwede: Toward the estimation of the absolute quality of individual protein structure models. Bioinformatics 27 (2011), 343-350. doi:10.1093/bioinformatics/btq662

29. Studio, BD: Dassault Systèmes BIOVIA. Discovery Studio Modeling Environment [Internet].; 2016.

30. Sleigh, BC, B Manna: Lymphedema. StatPearls Publishing, Treasure Island (FL).

31. Michelini, S, S Paolacci, E Manara, et al: Genetic tests in lymphatic vascular malformations and lymphedema. J. Med. Genet. 55 (2018), 222-232. doi:10.1136/jmedgenet-2017-105064

32. Laue, K, HM Pogoda, PB Daniel, et al: Craniosynostosis and multiple skeletal anomalies in humans and zebrafish result from a defect in the localized degradation of retinoic acid. Am. J. Hum. Genet. 89 (2011), 595-606. doi:10.1016/j.ajhg.2011.09.015

33. Kichaev, G, G Bhatia, PR Loh, et al: Leveraging polygenic functional enrichment to improve GWAS power. Am. J. Hum. Genet. 104 (2019), 65-75. doi:10.1016/j.ajhg.2018.11.008

34. Paolacci, S, A Zulian, A Bruson, et al: Genetic testing and therapeutic approaches. 2019. doi:10.23736/S0392-9590.19.04154-3

35. Mammadova, A, H Zhou, CEL Carels, et al: Retinoic acid signalling in the development of the epidermis, the limbs and the secondary palate. Differentiation 92 (2016), 326-335. doi:10.1016/j. diff.2016.05.001
Mirko Baglivo, MS
Magi Euregio
via Maso della Pieve 60/A
39100 Bolzano (BZ)
E-mail: mirko.baglivo@assomagi.org 